\title{
An Initiative to Improve 30-Day Readmission Rates Using a Transitions-of-Care Clinic Among a Mixed Urban and Rural Veteran Population
}

\author{
Benjamin R Griffin, MD1,2*, Neeru Agarwal, MD², Rachana Amberker, MBBS ${ }^{1,2}$, Jeydith A Gutierrez Perez, MD ${ }^{1,2}$, \\ Kelsi Eichorst, RN¹, Jennifer Chapin, MSN, RN ${ }^{1}$, Amy C Schweitzer, PharmD ${ }^{1}$, Mariko Hagiwara, MD 1,2, \\ Chaorong Wu, PhD ${ }^{3}$, Patrick Ten Eyck, PhD ${ }^{3}$, Heather Schacht Reisinger, PhD 1,2, Mary Vaughan-Sarrazin, PhD 1,2, \\ Ethan F Kuperman, $\mathrm{MD}^{2}$, Kevin Glenn, MD ${ }^{1,2}$, Diana I Jalal, MD ${ }^{1,2}$
}

1/owa City Veterans Affairs Health Care System, lowa City, lowa; ${ }^{2}$ Department of Medicine, University of lowa Carver College of Medicine, lowa City, lowa; Institute for Clinical and Translational Science, University of lowa, lowa City, lowa.

BACKGROUND/OBJECTIVE: Hospital readmissions in the United States, especially in patients at high-risk, cost more than $\$ 17$ billion annually. Although care transitions is an important area of research, data are limited regarding its efficacy, especially among rural patients. In this study, we describe a novel transitionsof-care clinic (TOCC) to reduce 30-day readmissions in a Veterans Health Administration setting that serves a high proportion of rural veterans.

METHODS: In this quality improvement initiative we conducted a pre-post study evaluating clinical outcomes in adult patients at high risk for 30-day readmission (Care Assessment Needs score > 85) discharged from the lowa City Veterans Affairs (ICVA) Health Care System from 2017 to 2020 . The ICVA serves 184,000 veterans across 50 counties in eastern lowa, western Illinois, and northern Missouri, with more than $60 \%$ of these patients residing in rural areas. We implemented a multidisciplinary TOCC to provide in-person or virtual follow-up to high-risk veterans after hospital discharge. The main purpose of this study was to assess how TOCC follow-up impacted the monthly 30-day patient readmission rate.

RESULTS: The TOCC resulted in a $19.2 \%$ relative reduction in 30-day readmission rates in the 12-month postimplementation period compared to the preimplementation period $(9.2 \%$ vs $11.4 \%, P=.04)$. Virtual visits were more popular than in-person visits among both urban and rural veterans. There was no difference in outcomes between these two follow-up options, and both groups had reduced readmission rates compared to nonTOCC follow-up.

CONCLUSIONS: A multidisciplinary TOCC within the ICVA featuring both virtual and in-person visits reduced the 30-day readmission rate. This reduction was particularly notable among patients with congestive heart failure. Journal of Hospital Medicine 2021;16:583-588.

(C) 2021 Society of Hospital Medicine ospital readmissions are a significant problem in the United States, affecting $15 \%$ to $30 \%$ of discharges and incurring costs of more than $\$ 17$ billion annually. ${ }^{1}$ Timely posthospitalization follow-up visits are critical to ensure the effective transfer of patients to the outpatient setting; such visits reduce readmission rates as well as hospital length of stay and overall healthcare resource utilization. ${ }^{2-4}$ Patients who receive inadequate follow-up care (ie, within 4 weeks of discharge) are significantly more likely to be readmitted than those who receive close follow-up care. ${ }^{5}$

Due to the large clinical and financial consequences associated with hospital readmission, a variety of interventions have been studied, including home visits, telemonitoring, medication management, telephone calls, and postdischarge clinics. ${ }^{6,7}$

*Corresponding Author: Benjamin R Griffin, MD;

Email: Benjamin-griffin@uiowa.edu; Telephone: 319-384-8197.

Published online first August 18, 2021.

Find additional supporting information in the online version of this article.

Received: February 9, 2021; Revised: May 19, 2021; Accepted: May 25, 2021

() 2021 Society of Hospital Medicine DOI 10.12788/jhm.3659
While studies have not shown postdischarge clinics to be universally efficacious in reducing readmission rates, there is increasing evidence of reduced readmission rates in clinics that target high-risk patients (eg, patients with congestive heart failure [CHF]) rather than the total population. ${ }^{2}$ A study by Hernandez et al that evaluated the relationship between early physician follow-up and 30-day readmissions showed a significantly lower readmission rate among hospitals with higher follow-up rates. ${ }^{8}$ Similarly, patients with CHF in a large, integrated health system who were seen within 7 days of discharge had an odds ratio (OR) of 0.81 (95\% Cl, 0.70-0.94) for 30-day readmissions. ${ }^{\text {? }}$

Transitions-of-care clinics (TOCC), designed to provide early postdischarge follow-up to high-risk patients, have been shown to reduce 30-day readmission rates, ${ }^{3,4,10,11}$ especially in clinics that have same-physician follow-up visits rather than follow-up visits with a community primary care physician (PCP). ${ }^{12}$ The most pronounced impact of postdischarge follow-up is seen in highrisk patients with high complexity or high severity of disease; however, complex rural patients are less likely to have access to specialty care. ${ }^{13}$ As a result, since rural residents must travel farther for specialty care, they are seen less frequently than their urban counterparts. ${ }^{14,15}$ 
Prior to our TOCC initiative, the lowa City VA (ICVA) ranked in the fifth quintile of the Veterans Health Administration (VHA) Strategic Analytics for Improvement and Learning model for hospital-wide readmissions (HWR), meaning that HWR at ICVA were higher than $80 \%$ of the other $\mathrm{VHA}$ healthcare centers. The low score in this metric was in part due to readmission rates in high-risk populations, including patients with $\mathrm{CHF}$ and those with high Care Assessment Need (CAN) scores. One concern was that the ICVA system serves many veterans from rural areas, some of whom must travel up to 200 miles to access inpatient and subspecialty care.

To meet these challenges, we implemented a TOCC to deliver timely postdischarge care focusing on high-risk and high-complexity patients. To address access-to-care issues of patients living in rural areas within the ICVA, we included virtual follow-up visits as a key component of our intervention. ${ }^{16,17}$ The aim of this project was to decrease 30-day readmission rates of ICVA patients by $20 \%$ within 12 months of implementation.

\section{METHODS}

\section{Setting/Study Population}

The ICVA serves 184,000 veterans stretched over 50 counties in eastern lowa, western Illinois, and northern Missouri, with more than $60 \%$ of these patients residing in rural areas. Patients were initially eligible for the TOCC if they had an admission diagnosis of CHF and a CAN score $>85$ at the time of discharge. The CAN score, developed by the VHA to assess the risk of hospital readmission in individual patients, factors in several variables, including demographics, coexisting conditions, vital signs, utilization of services, pharmacy visits, and laboratory results. Patients in the top 5\% (95-99) have a readmission rate of $20 \%$ at 90 days. Since the CAN is a proprietary tool, it may not be published in full; however, this assessment tool is commonly used and frequently cited in VA research. ${ }^{18-22}$ The CAN score is expressed as a percentile ranging from 0 (lowest risk) to 99 (highest risk). Patient eligibility was expanded during subsequent Plan-Do-Study-Act (PDSA) cycles, as outlined below. Patient eligibility was expanded during subsequent PDSA cycles (also outlined below). A review by a local institutional review board was obtained, and the study was classified as exempt due to the use of deidentified data. Standards for Quality Improvement Reporting Excellence 2.0 guidelines were used to construct the manuscript.

\section{Magnitude Assessment}

The numbers of discharges, readmissions within 30 days, emergency department (ED) visits by all discharged veterans, and veterans discharged with a CHF hospital diagnosis were recorded from February 2017 to February 2018, which were the 12 months immediately preceding the pilot implementation.

\section{Intervention}

The primary intervention was referral to the newly formed ICVA TOCC. The multidisciplinary TOCC team consisted of hospitalists, pharmacists, schedulers, and discharge planners/ care managers. Patients were identified by the hospitalist team during admission; prior to hospital discharge, these patients were referred to TOCC discharge planners to schedule appropriate follow-up appointments. Virtual follow-up visits were conducted using a patient's home technology; in cases where a patient lacked adequate technology capabilities (eg, no computer or internet access), the ICVA provided a tablet device with cellular internet capability for temporary use. Specific clinical activities included medication reconciliation by a pharmacist, follow-up of pending laboratory studies, imaging studies, pathology results, medical diagnosis education, counseling regarding dietary restrictions, and contingency planning outside of an ED visit in the event of a change in clinical status. In addition, the TOCC aimed to facilitate a smooth transition of care back to the PCP by arranging follow-up appointments, providing visit summaries, and scheduling consults with specialty care, as appropriate.

\section{Measures}

The primary objective measure was the 30-day readmission rate in the ICVA hospital. Secondary measures included the number of VA hospital ED visits within 30 days of discharge. The main process measures were the number of hospital discharges per month, the number of TOCC referrals, the number of TOCC appointments made, the number of virtual and in-person visits, and the percentage of appointment "no-shows."

\section{Implementation}

The TOCC was piloted from April 2018 to October 2018. During the pilot phase, TOCC enrollment was limited to virtual appointments and to patients with an admission diagnosis of CHF and a CAN score of $>85$. The TOCC had staff on-site 2 days a week; this included pharmacists to reconcile medications and hospitalists to address follow-up care needs.

The TOCC clinic was temporarily closed at the end of October 2018 to analyze pilot results. Based on stakeholder feedback, changes made as part of the second PDSA cycle included expanding eligibility criteria to any hospital admission diagnosis and to patients with a CAN score $<85$ if the hospitalist team felt the patient was likely to benefit from TOCC follow-up. In addition, on-site clinic staffing was expanded from 2 to 5 days per week to improve access, and the option for an in-person visit was added based on concerns some veterans expressed regarding the use of the technology at home. Finally, a formal resident program was added, and the order set for referrals was simplified. The TOCC was restarted in February 2019, and TOCC metrics were reviewed monthly. By July 2019, we identified issues with TOCC referrals and appointment creation that required additional modifications to the intervention.

A third PDSA cycle was initiated in July 2019 and included major changes, notably the formation of a designated TOCC committee. The committee appointed a dedicated TOCC scheduler whose role was to reduce confusion regarding scheduling, to update the discharge instructions/orders template to lower incidences of "double-booking" that occurred with PCP and TOCC appointments, to modify discharge educational instruction regarding virtual visits and tablet use, to 


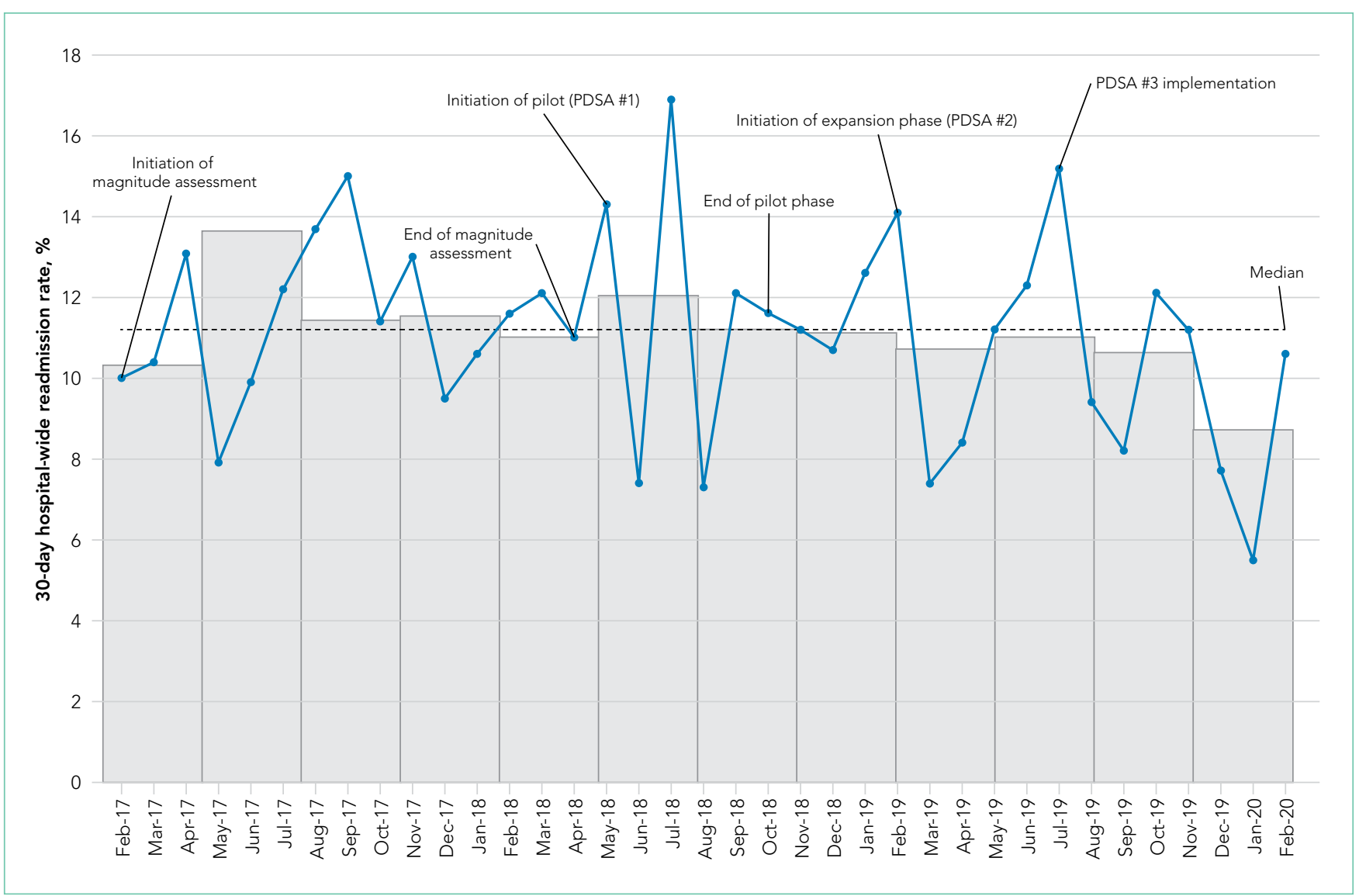

FIG. Run Chart Showing 30-Day Readmission Rates by Month Over Time in the Total Hospital Population. The gray bars represent the quarterly 30-day readmission rate. The dashed line is the median 30-day readmission rate for the 12-month intervention period.

adjust the TOCC-PCP handoff, and to formalize interactions between discharge coordinators and residents to review possible referrals every morning (Appendix Figure 1).

\section{Statistical Analysis}

Run charts were constructed by plotting monthly primary outcome values and monthly process metrics (Figure, Appendix Figure 2, Appendix Figure 3). Chi-square tests were used to compare 30-day readmission rates before and after the intervention. Statistical modeling was used to determine differences in outcomes between referred patients seen and referred patients not seen by the TOCC. In these statistical models, the outcome measures were 30-day readmissions, 30-day ED visits, and 6-month mortality. Covariates included in the final analysis were age, gender, race, CAN score, rural-urban commuting area code, referral service (resident vs nonresident), and admission diagnosis. Admission diagnoses were sorted by the investigators into one of the following seven categories: cardiac, infectious, pulmonary, gastrointestinal, neurologic, renal, and other.

Mean (SD) or counts and percentages were used to describe the distribution of continuous and categorical variables, respectively. Kruskal-Wallis test, $t$ test, or chi-square tests were used, as appropriate, across categories. Generalized linear models with a logistic link function were used to test for dif- ferences between patients who kept their appointment at the TOCC and those who did not keep their TOCC appointment (both unadjusted and adjusted for all of the covariates previously mentioned). In addition, generalized linear models were used to compare outcomes between TOCC patients seen virtually vs those seen in-person (both unadjusted and adjusted for all the covariates previously mentioned). All statistical tests were considered significant at a two-sided $P<.05$. All analyses were performed using SAS software version 9.4 (SAS Institute Inc).

\section{RESULTS}

\section{Magnitude Assessment}

During the preimplementation period (February 2017-February 2018), there were 3014 patient discharges from ICVA and 343 readmissions, resulting in a readmission rate of $11.4 \%$. Among patients with a hospital-admission diagnosis of cardiorespiratory disease, which included patients with $\mathrm{CHF}$, there were 381 discharges and 46 readmissions, resulting in a readmission rate of $12.1 \%$.

\section{Primary Outcome}

During the pilot phase, which was conducted from April 2018 to October 2018, 142 patients who met inclusion criteria (CHF diagnosis and a CAN score > 85) were discharged from ICVA, 
TABLE 1. Characteristics of Patients Referred to the Transitions-of-Care Clinic According to Whether They Were Seen or Not Seen and According to Visit Modality

\begin{tabular}{|c|c|c|c|c|c|c|}
\hline Characteristic & $\begin{array}{c}\text { Seen } \\
(n=240)\end{array}$ & $\begin{array}{l}\text { Not seen } \\
(n=247)\end{array}$ & $P$ value & $\begin{array}{l}\text { In-person visit } \\
\quad(\mathrm{n}=82)\end{array}$ & $\begin{array}{l}\text { Virtual visit } \\
(n=158)\end{array}$ & $P$ value \\
\hline Age, mean (SD), y & $68(12)$ & $67(11)$ & .76 & $69(10)$ & $68(12.1)$ & .51 \\
\hline Male, №. (\%) & $235(97)$ & $238(96)$ & .30 & $80(97)$ & $155(98)$ & .78 \\
\hline Race, No. (\%) & & & .31 & & & .14 \\
\hline White & $218(91)$ & $233(93)$ & & $74(90)$ & $144(91)$ & \\
\hline Black & $8(3)$ & $6(3)$ & & $5(6)$ & $3(2)$ & \\
\hline Other & $14(6)$ & $9(4)$ & & $3(4)$ & $11(7)$ & \\
\hline CAN score, mean (SD) & $95(12)$ & $97(9)$ & $<.001$ & $95(13)$ & $90(13)$ & .82 \\
\hline Rural RUCA score, mean (SD) & $125(52)$ & $131(54)$ & .76 & $41(50)$ & $84(54)$ & .54 \\
\hline Primary admission diagnosis, №. (\%) & & & .30 & & & .19 \\
\hline Cardiac & $78(33)$ & $76(31)$ & & $29(35)$ & $49(31)$ & \\
\hline Infectious & $36(15)$ & $42(17)$ & & $10(12)$ & $26(16)$ & \\
\hline Pulmonary & $37(15)$ & $32(13)$ & & $6(7)$ & $31(20)$ & \\
\hline Gastrointestinal & $36(15)$ & $42(17)$ & & $13(16)$ & $20(13)$ & \\
\hline Neurology & $17(7)$ & $18(7)$ & & $7(9)$ & $10(6)$ & \\
\hline Renal & $15(6)$ & $13(5)$ & & $6(7)$ & $9(6)$ & \\
\hline Other & $24(10)$ & $24(10)$ & & $11(13)$ & $13(8)$ & \\
\hline 30-day readmissions, №. (\%) & $23(9.6)$ & $68(27.8)$ & $<.001$ & $8(9.5)$ & $15(9.9)$ & .92 \\
\hline ED visits, No. (\%) & $36(15.0)$ & $80(32.9)$ & $<.001$ & $12(14.6)$ & $24(15.2)$ & .91 \\
\hline
\end{tabular}

Abbreviations: CAN, Care Assessment Needs; ED, emergency department; RUCA, rural-urban commuting areas.

and 56 referrals to the TOCC were placed. The readmission rate among the cardiorespiratory cohort of veterans was $9.5 \%$.

During the expansion of the intervention from February 2019 to February 2020, there were 2844 discharges from the ICVA and 291 readmissions, resulting in a readmission rate of $10.2 \%$. However, there was a further decrease in the readmission rate after the third PDSA cycle was initiated in July 2019 (Appendix Figure 1). The readmission rate was $9.2 \%$ in the final 6 months of the intervention period, and $7.9 \%$ in the final 3 months. Of note, in the group of 1948 patients who did not meet the eligibility criteria to participate in our study, the readmission rate during the same time period was $8.6 \%$ (161 readmissions).

When comparing the 6 months following the third PDSA cycle to the magnitude assessment period, there was a relative readmission reduction of $19.3 \%(P=.04)$, and an absolute reduction of $2.2 \%$. If the final 3 months of the intervention period are included, there was an absolute reduction of $3.5 \%$ and a relative reduction of $30.7 \%(P=.01)$. Notably, before the pilot phase, ICVA was in the fifth quintile for HWR among VA hospitals but improved to the second quintile by the end of the expansion phase.

\section{Process Outcomes}

Process metrics for TOCC referrals, the number of patients seen, and the number of virtual and in-person visits over time are shown in Appendix Figure 3. Rates of TOCC referrals and the number of TOCC visits were lower than anticipated during the first 5 months of the intervention. However, TOCC referrals increased significantly after we implemented the previously described changes as part of the third PDSA cycle. As a result, total, virtual, and in-person visits also significantly increased from July 2019 to February 2020. The proportion of patients choosing virtual vs in-person visits fluctuated over time, but virtual visits were generally chosen more often than in-person visits.

\section{Statistical Modeling \\ Baseline Data}

Cohort characteristics are shown in Table 1. The cohort, which reflected the ICVA population, was predominantly male (96\%) and White (93\%), with a mean age of 67 years. The population was approximately half urban and half rural in composition, and the most common reason for hospital admission was cardiac. Other than a small but statistically significant difference in CAN scores, there were no significant differences between patients who kept their TOCC appointment and those who did not. There were also no differences in baseline characteristics between patients who chose virtual follow-up and patients who chose in-person follow-up, including the proportion of urban and rural patients.

\section{Outcomes}

Patients who kept their TOCC appointments had a 30-day readmission rate of $9.6 \%$, which was significantly lower than the 30 day readmission rate of $27 \%$ in the group that did not keep their 
TOCC appointment $(P<.001)$. Similarly, the percentage of patients treated in the ED was 15\% in the TOCC group compared to $31.2 \%$ in the group that canceled their appointment $(P<.001)$ (Table 1). In the multivariable analysis, patients who were seen in the TOCC group had an OR for 30-day readmission of 0.35 (95\% $\mathrm{Cl}, 0.19-0.62, P<.001)$, and an OR for ED visits of $0.39(95 \% \mathrm{Cl}$, $0.23-0.65 ; P<.001$ ) (Table 2). There was no statistically significant difference in 6-month mortality between the two groups. In the virtual group compared to the in-person group, there were no statistically significant differences in outcomes between the two groups in the unadjusted or adjusted analysis (Table 2).

\section{DISCUSSION}

In this quality improvement initiative, we found that a TOCC targeting high-risk patients and offering virtual follow-up visits significantly decreased the 30-day readmission rates among veterans at ICVA. Statistical comparisons of patients seen at the TOCC vs those not seen at the TOCC showed a dramatic reduction in 30-day readmissions and ED visits. Finally, virtual follow-ups were more popular than in-person visits, and patients who followed up virtually had equivalent outcomes to those with the more traditional follow-up.

In the expansion phase, eligibility was expanded to include any hospital indication but continued to focus on high-risk patients. Existing literature suggests that providing postdischarge care to all patients, including low- or medium-risk patients, may not be as impactful as enrolling high-risk patients only. For instance, a postdischarge clinic offered to all patients at a VA system in Colorado did not reduce readmission rates compared to PCP follow-up. ${ }^{23}$ In contrast, a study of more than 10,000 high-risk urban patients demonstrated that postdischarge care resulted in a $9.3 \%$ reduction in readmission risk. ${ }^{24}$ Our data are consistent with the previously published studies, as the average CAN score of patients seen in TOCC was 90 , suggesting a high risk of readmission. In the final 12 months of the intervention, 15\% of discharged patients were seen at the TOCC clinic, suggesting that targeted intervention within the small subset of high-risk patients was sufficient to achieve our primary aim. Of note, among patients who did not meet the inclusion criteria for TOCC referral (ie, patients not considered high risk [CAN score $\leq 85]$ ]), the rate of readmissions was $8.6 \%$.

Most of the available research on the efficacy of postdischarge clinics was conducted in urban environments. Our ICVA population sees a large proportion of rural veterans, who account for just over $50 \%$ of the discharge population. In a study of more than 2 million Medicare patients discharged from US hospitals, the 30-day readmission rates and adjusted mortality rates were higher among patients in rural counties, and post-acute care seemed to have a greater impact in rural rather than urban settings. ${ }^{25}$ Previous studies have demonstrated that virtual visits have the potential to improve readmission rates, especially in patients with $\mathrm{CHF}^{26}$ and in patients at the highest risk for readmission. ${ }^{27}$ In our study, the pilot phase offered only virtual visits, but we subsequently added an in-person option based on veteran feedback. Interestingly, over the next 12 months, virtual
TABLE 2. Unadjusted and Adjusted Primary and Secondary Outcomes of Patients Referred to Transitions-of-Care Clinic According to Whether They Were Seen or Not Seen and According to Visit Modality

\begin{tabular}{|c|c|c|c|c|}
\hline \multirow[b]{2}{*}{ Outcome } & \multicolumn{2}{|c|}{ TOCC seen vs canceled ${ }^{a}$} & \multicolumn{2}{|c|}{$\begin{array}{l}\text { In-person visit } \\
\text { vs virtual visit }\end{array}$} \\
\hline & OR $(95 \% \mathrm{Cl})$ & $P$ value & OR $(95 \% \mathrm{Cl})$ & $P$ value \\
\hline \multicolumn{5}{|l|}{ 30-day readmission } \\
\hline Unadjusted & $0.28(0.17-0.46)$ & $<.001$ & $1.04(0.42-2.58)$ & .92 \\
\hline Multivariable adjusted & $0.35(0.19-0.62)$ & $<.001$ & $0.83(0.26-2.63)$ & .90 \\
\hline \multicolumn{5}{|l|}{ 30-day ED visit } \\
\hline Unadjusted & $0.36(0.23-0.56)$ & $<.001$ & $0.96(0.45-2.03)$ & .91 \\
\hline Multivariable adjusted & $0.39(0.23-0.65)$ & $<.001$ & $0.73(0.26-2.00)$ & .53 \\
\hline \multicolumn{5}{|l|}{ 6-month mortality } \\
\hline Unadjusted & $0.82(0.48-1.41)$ & .48 & $1.37(0.61-3.12)$ & .45 \\
\hline Multivariable adjusted & $0.65(0.33-1.28)$ & .21 & $0.82(0.23-2.93)$ & .75 \\
\hline \multicolumn{5}{|c|}{ aSample size was 484 ( $n=239$ vs 245). } \\
\hline Abbreviations: ED, emer & gency department; & $\mathrm{OCC}$, trans & ons-of-care clinic. & \\
\hline
\end{tabular}

visits were more popular with both urban and rural veterans, and there were no differences in the number of rural patients in the in-person vs the virtual group. These findings suggest factors other than rurality influenced the decision to choose virtual follow-up visits over in-person visits. Future studies should seek to determine the extent to which factors such as age, race, educational level, and socioeconomic circumstances impact veterans' follow-up decisions. Not only were outcomes among patients who chose virtual visits the same as those of patients who chose in-person visits, but both of these groups had better outcomes compared to the non-TOCC group (Table 2). This finding demonstrating the efficacy of virtual visits among rural and urban patients has taken on increased significance due to the COVID-19 pandemic, as virtual visits offer a safer option, one that minimizes physical contact.

Our quality improvement analysis included a statistical comparison of patients seen vs those not seen at the TOCC. Patients who were referred to the TOCC but chose not to keep their appointment were similar to those seen in TOCC in terms of age, CAN score, rurality, and hospital diagnosis, but readmission rates were substantially higher in this group even after adjustments for covariates (Table 2). Evaluating causality in interventions aimed to reduce hospital readmission rates is complicated. ${ }^{28}$ Our findings add greater plausibility to the utility of TOCC in accounting for at least a portion of the reported decrease in ICVA 30-day readmissions.

Our study has several strengths, including an observation period longer than 2 years, a large population of discharged veterans within an integrated healthcare system, and a large 
proportion of patients living in rural areas. Another strength of our study is the innovative nature of the intervention, which features a multidisciplinary team and the option of virtual or in-person visits. Nevertheless, this study also has several important limitations. As a single-center study, our findings may not be generalizable to other institutions, especially those outside the VHA system. Similarly, our study population reflected that of the ICVA, which may limit generalizability to a more diverse population. While we attempted to account in our statistical modeling for baseline differences between referred patients seen by the TOCC and those referred but not seen, we cannot exclude residual confounding between the groups. Specifically, the comparison of patients who did and did not choose TOCC follow-up introduces the possibility of selection bias. Future randomized/controlled studies will need to evaluate whether TOCC is more effective than the standard of care to reduce readmissions. Finally, since the analysis period following the final PDSA cycle was compressed due to the onset of the COVID-19 pandemic in the United States, no data are available regarding the sustained impacts of changes made during this cycle.

\section{CONCLUSION}

A multidisciplinary TOCC within the ICVA, featuring both virtual and in-person visits, reduced 30-day readmission rates by $19.3 \%$; this approach to care was especially effective in patients with CHF. Virtual visits were the follow-up mode of choice for both urban and rural veterans, and there was no difference in outcomes between these two follow-up options. Future studies will focus on additional quality metrics, including costeffectiveness and patient satisfaction.

Disclosures: The authors reported no conflicts of interest.

Funding: This study was supported in part by The University of lowa Clinical and Translational Science Award granted with funds from the National Institutes of Health (UL1TR002537).

\section{References}

1. Jencks SF, Williams MV, Coleman EA. Rehospitalizations among patients in the Medicare fee-for-service program. N Engl J Med. 2009;360(14):14181428. https://doi.org/10.1056/nejmsa0803563

2. Doctoroff L. Postdischarge clinics and hospitalists: a review of the evidence and existing models. J Hosp Med. 2017;12(6):467-471. https://doi. org/10.12788/jhm.2750

3. Koehler BE, Richter KM, Youngblood L, et al. Reduction of 30-day postdischarge hospital readmission or emergency department (ED) visit rates in high-risk elderly medical patients through delivery of a targeted care bundle. J Hosp Med. 2009;4(4):211-218. https://doi.org/10.1002/jhm.427

4. Abrashkin KA, Cho HJ, Torgalkar S, Markoff B. Improving transitions of care from hospital to home: what works? Mt Sinai J Med. 2012;79(5):535-544. https://doi.org/10.1002/msj.21332

5. Misky GJ, Wald HL, Coleman EA. Post-hospitalization transitions: examining the effects of timing of primary care provider follow-up. J Hosp Med. 2010;5(7):392-397. https://doi.org/10.1002/jhm.666

6. Greysen SR, Harrison JD, Kripalani S, et al. Understanding patient-centred readmission factors: a multi-site, mixed-methods study. BMJ Qual Saf. 2017;26(1):33-41. https://doi.org/10.1136/bmjqs-2015-004570

7. Hansen LO, Young RS, Hinami K, Leung A, Williams MV. Interventions to reduce 30-day rehospitalization: a systematic review. Ann Intern Med. 2011;155(8):520-528. https://doi.org/10.7326/0003-4819-155-8-20111018000008
8. Hernandez AF, Greiner MA, Fonarow GC, et al. Relationship between early physician follow-up and 30-day readmission among Medicare beneficiaries hospitalized for heart failure. JAMA. 2010;303(17):1716-1722. https://doi.org/10.1001/ jama.2010.533

9. Lee KK, Yang J, Hernandez AF, Steimle AE, Go AS. Post-discharge follow-up characteristics associated with 30-day readmission after heart failure hospitalization. Med Care. 2016;54(4):365-372. https://doi.org/10.1097/ mlr.0000000000000492

10. Balaban RB, Williams MV. Improving care transitions: hospitalists partnering with primary care. J Hosp Med. 2010;5(7):375-377. https://doi.org/10.1002/ jhm.824

11. Rodrigues $C R$, Harrington AR, Murdock N, et al. Effect of pharmacy-supported transition-of-care interventions on 30-day readmissions: a systematic review and meta-analysis. Ann Pharmacother. 2017;51(10):866-889. https:// doi.org/10.1177/1060028017712725

12. van Walraven $C$, Taljaard M, Etchells $E$, et al. The independent association of provider and information continuity on outcomes after hospital discharge: implications for hospitalists. J Hosp Med. 2010;5(7):398-405. https://doi.org/10.1002/ jhm.716

13. Gruca TS, Pyo TH, Nelson GC. Providing cardiology care in rural areas through vsiting consultant clinics. J Am Heart Assoc. 2016;5(7):e002909. https://doi.org/10.1161/jaha.115.002909

14. Chan L, Hart LG, Goodman DC. Geographic access to health care for rural Medicare beneficiaries. J Rural Health. 2006;22(2):140-146. https:// doi.org/10.1111/j.1748-0361.2006.00022.x

15. Burke RE, Jones CD, Coleman EA, Falvey JR, Stevens-Lapsley JE, Ginde AA. Use of post-acute care after hospital discharge in urban and rural hospitals. Am J Accountable Care. 2017;5(1):16-22.

16. Jetty A, Moore MA, Coffman M, Petterson S, Bazemore A. Rural family physicians are twice as likely to use telehealth as urban family physicians. Telemed J E Health. 2018;24(4):268-276. https://doi.org/10.1089/tmj.2017.0161

17. Harrison PL, Hara PA, Pope JE, Young MC, Rula EY. The impact of postdischarge telephonic follow-up on hospital readmissions. Popul Health Manag. 2011;14(1):27-32. https://doi.org/10.1089/pop.2009.0076

18. Wang L, Porter B, Maynard C, et al. Predicting risk of hospitalization or death among patients receiving primary care in the Veterans Health Administration. Med Care. 2013;51(4):368-373. https://doi.org/10.1097/mlr.0b013e31827da95a

19. Spece LJ, Donovan LM, Griffith MF, et al. Initiating low-value inhaled corticosteroids in an inception cohort with chronic obstructive pulmonary disease. Ann Am Thorac Soc. 2020;17(5):589-595. https://doi.org/10.1513/annalsats.201911-8540c

20. Osborne TF, Suarez P, Edwards D, Hernandez-Boussard T, Curtin C. Patient electronic health records score for preoperative risk assessment before total knee arthroplasty. JB JS Open Access. 2020;5(2):e0061. https://doi.org/10.2106/jbjs. oa.19.00061

21. Levy $C$, Ersek M, Scott W, et al. Life-sustaining treatment decisions initiative: early implementation results of a national Veterans Affairs program to honor veterans' care preferences. J Gen Intern Med. 2020;35(6):1803-1812. https:// doi.org/10.1007/s11606-020-05697-2

22. Ibrahim SA. High-risk patients and utilization of primary care in the US Veterans Affairs health system. JAMA Netw Open. 2020;3(6):e209518. https:// doi.org/10.1001/jamanetworkopen.2020.9518

23. Burke RE, Whitfield E, Prochazka AV. Effect of a hospitalist-run postdischarge clinic on outcomes. J Hosp Med. 2014;9(1):7-12. https://doi.org/10.1002/ jhm.2099

24. Jeng GY, Doyle MM, Belton BM, Herrin J, Horwitz LI. Quasi-experimental evaluation of the effectiveness of a large-scale readmission reduction program. JAMA Intern Med. 2016;176(5):681-690. https://doi.org/10.1001/ jamainternmed.2016.0833

25. Kosar CM, Loomer L, Ferdows NB, Trivedi AN, Panagiotou OA, Rahman M. Assessment of rural-urban differences in postacute care utilization and outcomes among older US adults. JAMA Netw Open. 2020;3(1):e1918738. https://doi.org/10.1001/jamanetworkopen.2019.18738

26. Pandor A, Thokala P, Gomersall T, et al. Home telemonitoring or structured telephone support programmes after recent discharge in patients with heart failure: systematic review and economic evaluation. Health Technol Assess. 2013;17(32):1-207, v-vi. https://doi.org/10.3310/hta17320

27. Low LL, Tan SY, Ng MJM, et al. Applying the integrated practice unit concept to a modified virtual ward model of care for patients at highest risk of readmission: a randomized controlled trial. PloS One. 2017;12(1):e0168757. https://doi.org/10.1371/journal.pone.0168757

28. Mcllvennan CK, Eapen ZJ, Allen LA. Hospital readmissions reduction program. Circulation. 2015;131(20):1796-1803. https://doi.org/10.1161/ circulationaha.114.010270 